\title{
About the spectra of a real nonnegative matrix and its signings
}

\author{
K. Attas, A. Boussaïri*, and M. Zaidi
}

Communicated by Yu. A. Drozd

\begin{abstract}
For a complex matrix $M$, we denote by $\operatorname{Sp}(M)$ the spectrum of $M$ and by $|M|$ its absolute value, that is the matrix obtained from $M$ by replacing each entry of $M$ by its absolute value. Let $A$ be a nonnegative real matrix, we call a signing of $A$ every real matrix $B$ such that $|B|=A$. In this paper, we characterize the set of all signings of $A$ such that $\operatorname{Sp}(B)=\alpha \operatorname{Sp}(A)$ where $\alpha$ is a complex unit number. Our motivation comes from some recent results about the relationship between the spectrum of a graph and the skew spectra of its orientations.
\end{abstract}

\section{Introduction}

Throughout this paper, all matrices are complex, unless otherwise noted. The identity matrix of order $n$ is denoted by $I_{n}$ and the transpose of a matrix $A$ by $A^{T}$. Let $\Sigma$ be a subgroup of $\mathbb{C}^{*}$, the group of nonzero complex numbers under multiplication. Two square matrices $A$ and $B$ are $\Sigma$ diagonally similar if $B=\Lambda^{-1} A \Lambda$ for some diagonal matrix $\Lambda$ with diagonal entries in $\Sigma$. A square matrix $A$ is reducible if there exists a permutation matrix $P$, so that $A$ can be reduced to the form $P A P^{T}=\left(\begin{array}{cc}X & Y \\ 0 & Z\end{array}\right)$ where $X$ and $Z$ are square matrices. A square matrix which is not reducible is

${ }^{*}$ Corresponding author.

2020 MSC: 05C20, 05C50.

Key words and phrases: spectra, digraphs, nonnegative matrices, irreducible matrices. 
said to be irreducible. A real matrix $A$ is nonnegative, (we write $A \geqslant 0$ ), if all its entries are nonnegative.

Let $A$ be an $n \times n$ real or complex matrix. The multiset $\left\{\lambda_{1}, \ldots, \lambda_{n}\right\}$ of eigenvalues of $A$ is called the spectrum of $A$ and is denoted by $\operatorname{Sp}(A)$. We usually assume that $\left|\lambda_{1}\right| \geqslant\left|\lambda_{2}\right| \geqslant \ldots \geqslant\left|\lambda_{n}\right|$. The spectral radius of $A$ is $\rho(A):=\left|\lambda_{1}\right|$.

The relationship between the spectrum of a graph and the skew spectra of its orientations is studied in many papers (see for example $[1,2,4-6,8,10]$ ). Our work is closely related to the result of Shader and So [8]. To state this result, we need to introduce some definitions and notations.

Let $G$ be a finite simple graph with vertex set $V(G)=\left\{v_{1}, \ldots, v_{n}\right\}$ and edge set $E(G)$. The adjacency matrix of $G$ is the symmetric matrix $A(G)=\left(a_{i j}\right)_{1 \leqslant i, j \leqslant n}$ where $a_{i j}=a_{j i}=1$ if $\left\{v_{i}, v_{j}\right\}$ is an edge of $G$ and $a_{i j}=a_{j i}=0$ otherwise. Since the matrix $A(G)$ is symmetric, its eigenvalues are real. The adjacency spectrum $\operatorname{Sp}(G)$ of $G$ is defined as the spectrum of $A(G)$. Let $G^{\sigma}$ be an orientation of $G$, which assigns to each edge a direction so that the resultant graph $G^{\sigma}$ becomes an oriented graph. The skew-adjacency matrix of $G^{\sigma}$ is the real skew-symmetric matrix $S\left(G^{\sigma}\right)=\left(a_{i j}^{\sigma}\right)_{1 \leqslant i, j \leqslant n}$ where $a_{i j}^{\sigma}=-a_{j i}^{\sigma}=1$ if $\left(v_{i}, v_{j}\right)$ is an arc of $G^{\sigma}$ and $a_{i j}^{\sigma}=0$ otherwise. The skew-spectrum $\operatorname{Sp}\left(G^{\sigma}\right)$ of $G^{\sigma}$ is defined as the spectrum of $S\left(G^{\sigma}\right)$. Note that $\operatorname{Sp}\left(G^{\sigma}\right)$ consists of only purely imaginary eigenvalues because $S\left(G^{\sigma}\right)$ is a real skew-symmetric matrix.

Let $G$ be a bipartite graph with bipartition $[I, J]$, the orientation $G^{\varepsilon}$ that assigns to each edge of $G$ a direction from $I$ to $J$ is called the canonical orientation. Shader and So [8] showed that $\operatorname{Sp}\left(G^{\varepsilon}\right)=i \operatorname{Sp}(G)$. Moreover, they proved that a graph $G$ is bipartite if and only if $\operatorname{Sp}\left(G^{\sigma}\right)=i \operatorname{Sp}(G)$ for some orientation $G^{\sigma}$ of $G$.

Consider now two orientations $G^{\sigma}$ and $G^{\tau}$ of $G$. We say that $G^{\sigma}$ and $G^{\tau}$ are switching-equivalent if there exists a subset $W$ of $V(G)$ such that $G^{\sigma}$ is obtained from $G^{\tau}$ by reversing the direction of all arcs between $W$ and $V(G) \backslash W$. Clearly, the skew-adjacency matrices of switchingequivalent orientations are $\{-1,1\}$-diagonally similar. Hence, they have the same spectrum. When $G$ is bipartite, Anuradha et al. [1] proved that $\operatorname{Sp}\left(G^{\sigma}\right)=i \operatorname{Sp}(G)$ if and only if $G^{\sigma}$ is switching-equivalent to the canonical orientation.

These results can be stated in term of matrices as follows.

Proposition 1. Let $A$ be a $\{0,1\}$-symmetric matrix. Then the following statements are equivalent: 
i) There exists a real skew-symmetric matrix $B$ such that $|B|=A$ and $\operatorname{Sp}(B)=i \operatorname{Sp}(A)$;

ii) There exists a permutation matrix $P$ such that

$$
P A P^{T}=\left(\begin{array}{cc}
0 & X \\
X^{T} & 0
\end{array}\right)
$$

where the zero diagonal blocks are square.

Proposition 2. Let $A=\left(\begin{array}{cc}0 & X \\ X^{T} & 0\end{array}\right)$ be a $\{0,1\}$-symmetric matrix and let $B$ be a skew-symmetric matrix such that $|B|=A$. Then, the following statements are equivalent:

i) $\operatorname{Sp}(B)=i \operatorname{Sp}(A)$;

ii) $B$ is $\{-1,1\}$-diagonally similar to $\widetilde{A}=\left(\begin{array}{cc}0 & X \\ -X^{T} & 0\end{array}\right)$.

For a $\{0,1\}$-symmetric matrix $A$, the propositions above characterize the set of all skew-symmetric signings $B$ of $A$, such that $\operatorname{Sp}(B)=i \operatorname{Sp}(A)$. In this paper, we consider the more general problem.

Problem 1. Let $A$ be a nonnegative real matrix and let $\alpha$ be a complex unit number. Characterize the set of all signings $B$ of $A$ such that $\operatorname{Sp}(B)=$ $\alpha \operatorname{Sp}(A)$.

We solve this problem when $A$ is an irreducible matrix. To state our main result, we need some terminology. A digraph $D$ is a pair consisting of a finite set $V(D)$ of vertices and a subset $E(D)$ of ordered pairs of vertices called arcs. Let $v, v^{\prime}$ be two vertices of $D$, a path $P$ from $v$ to $v^{\prime}$ is a finite sequence $v_{0}=v, \ldots, v_{k}=v^{\prime}$ such that $\left(v_{0}, v_{1}\right), \ldots,\left(v_{k-1}, v_{k}\right)$ are arcs of $D$. The length of $P$ is the number $k$ of its arcs. If $v_{0}=v_{k}$, we say that $P$ is a closed path. A digraph is said to be strongly connected if for any two vertices $v$ and $v^{\prime}$, there is a path joining $v$ to $v^{\prime}$. It is easy to see that a strongly connected digraph contains a closed path. The period of a strongly connected digraph is the greatest common divisor of the lengths of its closed paths.

With each $n \times n$ matrix $A=\left(a_{i j}\right)_{1 \leqslant i, j \leqslant n}$, we associate a digraph $D_{A}$ on the vertex set $[n]=\{1, \ldots, n\}$ and with arc set $\left\{(i, j): a_{i j} \neq 0\right\}$. It is easy to show that $A$ is irreducible if and only if $D_{A}$ is strongly connected. The period of an irreducible matrix is the period of its associate digraph. For example, if $A$ is the adjacency matrix of a connected graph $G$, then 
its period is either 1 or 2 . Moreover, the period of $A$ is 2 if and only if $G$ is bipartite.

Let $A$ be an irreducible nonnegative real matrix of period $p$. For each complex unit number $\alpha$, we denote by $\mathcal{M}(\alpha, A)$ the set of all signings $B$ of $A$ such that $\operatorname{Sp}(B)=\alpha \operatorname{Sp}(A)$.

In Corollary 2, we prove that if $\mathcal{M}(\alpha, A)$ is nonempty, then $\alpha=e^{\frac{i \pi k}{p}}$ for some $k \in\{0, \ldots, 2 p-1\}$. Moreover, we prove in Proposition 6 that $e^{\frac{i 2 \pi}{p}} \operatorname{sp}(A)=\operatorname{sp}(A)$. This implies that,

$$
\begin{aligned}
\mathcal{M}(1, A) & =\mathcal{M}\left(e^{\frac{2 i \pi}{p}}, A\right)=\cdots=\mathcal{M}\left(e^{\frac{2(p-1) i \pi}{p}}, A\right) \\
\mathcal{M}\left(e^{\frac{i \pi}{p}}, A\right) & =\mathcal{M}\left(e^{\frac{3 i \pi}{p}}, A\right)=\cdots=\mathcal{M}\left(e^{\frac{(2 p-1) i \pi}{p}}, A\right)
\end{aligned}
$$

Therefore, it suffices to characterize $\mathcal{M}(1, A)$ and $\mathcal{M}\left(e^{\frac{i \pi}{p}}, A\right)$.

In the proof of Corollary 1, we give an explicit construction of a matrix $B_{0} \in \mathcal{M}\left(e^{\frac{i \pi}{p}}, A\right)$ which is used in our main theorem below.

Theorem 1. Under the notation above, the following statements hold

i) $\mathcal{M}(1, A)$ is the set of matrices $\{-1,1\}$-diagonally similar to $A$;

ii) $\mathcal{M}\left(e^{\frac{i \pi}{p}}, A\right)$ is the set of matrices $\{-1,1\}$-diagonally similar to $B_{0}$.

\section{Some properties of $\mathcal{M}(\alpha, A)$}

Throughout, $A$ is an $n \times n$ irreducible nonnegative matrix, $p$ its period and $\alpha$ a unit complex number. We will use the following theorem due to Helmut Wielandt [9].

Theorem 2. Let $B$ be a complex $n \times n$ matrix such that $|B| \leqslant A$. Then $\rho(B) \leqslant \rho(A)$. Moreover, if equality holds (i.e., $\rho(A) e^{i \theta} \in \operatorname{Sp}(B)$ for some real number $\theta$ ) then $B=e^{i \theta} L A L^{-1}$, where $L$ is a complex diagonal matrix such that $|L|=I_{n}$.

We will use Theorem 2 to prove the following.

Proposition 3. Let $B$ be a signing of $A$ such that $\rho(B)=\rho(A)$. If $\lambda$ is an eigenvalue of $B$ such that $|\lambda|=\rho(A)$, then $\lambda=\rho(A) e^{\frac{i \pi k}{p}}$ for some $k \in\{0, \ldots, 2 p-1\}$.

Proof. Let $A:=\left(a_{i j}\right)_{1 \leqslant i, j \leqslant n}, B:=\left(b_{i j}\right)_{1 \leqslant i, j \leqslant n}$ and $\lambda=\rho(A) e^{i \theta}$. By Theorem 2, we have $B=e^{i \theta} L A L^{-1}$ where $L$ is a complex diagonal matrix such that $|L|=I_{n}$. It follows that $b_{i j}=e^{i \theta} l_{i} a_{i j} l_{j}^{-1}$ for $i, j \in\{1, \ldots, n\}$, 
where $l_{1}, \ldots, l_{n}$ are the diagonal entries of $L$. Consider now a closed path $C=\left(i_{1}, i_{2}, \ldots, i_{r}, i_{1}\right)$ of $D_{A}$. By the previous equality, we have

$$
\frac{b_{i_{1} i_{2}} \ldots b_{i_{r-1} i_{r}} b_{i_{r} i_{1}}}{a_{i_{1} i_{2}} \ldots a_{i_{r-1} i_{r}} a_{i_{r} i_{1}}}=\left(e^{i \theta} l_{i_{1}} l_{i_{2}}^{-1}\right) \ldots\left(e^{i \theta} l_{i_{r-1}} l_{i_{r}}^{-1}\right)\left(e^{i \theta} l_{i_{r}} l_{i_{1}}^{-1}\right)=\left(e^{i \theta}\right)^{r}
$$

Then $\left(e^{i \theta}\right)^{r} \in\{1,-1\}$ because $|B|=A$.

Since $p$ is the greatest common divisor of the lengths of the closed paths in $D_{A}$, we have $\left(e^{i \theta}\right)^{p} \in\{1,-1\}$ and then $\lambda=\rho(A) e^{\frac{i \pi k}{p}}$ for some $k \in\{0, \ldots, 2 p-1\}$.

Remark 1. Let $\lambda$ be an eigenvalue of $A$ such that $|\lambda|=\rho(A)$. By applying Proposition 3 to $B=A$, we have $\lambda=\rho(A) e^{\frac{i \pi k}{p}}$ for some $k \in$ $\{0, \ldots, 2 p-1\}$.

The following result gives a necessary condition under which $\mathcal{M}(\alpha, A)$ is nonempty.

Corollary 1. If $\mathcal{M}(\alpha, A)$ is nonempty then $\alpha=e^{\frac{i \pi k}{p}}$ for some $k \in$ $\{0, \ldots, 2 p-1\}$, or equivalently $\alpha^{p}= \pm 1$.

Proof. Let $\lambda$ be an eigenvalue of $A$ such that $|\lambda|=\rho(A)$. By Remark 1, we have $\lambda=\rho(A) e^{\frac{i \pi k}{p}}$ for some $k \in\{0, \ldots, 2 p-1\}$. Let $B \in \mathcal{M}(\alpha, A)$. Then $\alpha \rho(A) e^{\frac{i \pi k}{p}} \in \operatorname{Sp}(B)$ because $\operatorname{Sp}(B)=\alpha \operatorname{Sp}(A)$. It follows from Proposition 3 that $\alpha \rho(A) e^{\frac{i \pi k}{p}}=\rho(A) e^{\frac{i \pi h}{p}}$ for some $h \in\{0, \ldots, 2 p-1\}$ and hence $\alpha=e^{\frac{i \pi(h-k)}{p}}$.

It is easy to see that if $B \in \mathcal{M}(\alpha, A)$, then $\Lambda^{-1} B \Lambda \in \mathcal{M}(\alpha, A)$ for every $\{-1,1\}$-diagonal matrix $\Lambda$. Conversely,

Proposition 4. The matrices in the set $\mathcal{M}(\alpha, A)$ are all $\{-1,1\}$ diagonally similar.

Proof. Let $B_{1}, B_{2} \in \mathcal{M}(\alpha, A)$. Then $\operatorname{Sp}\left(B_{1}\right)=\operatorname{Sp}\left(B_{2}\right)=\alpha \operatorname{Sp}(A)$. It follows that $B_{1}$ and $B_{2}$ have a common eigenvalue of the form $\rho(A) e^{i \theta}$ for some real number $\theta$. By Theorem 2, we have $B_{1}=e^{i \theta} L_{1} A L_{1}^{-1}$ and $B_{2}=e^{i \theta} L_{2} A L_{2}^{-1}$ where $L_{1}, L_{2}$ are complex diagonal matrices such that $\left|L_{1}\right|=\left|L_{2}\right|=I_{n}$. It follows that $B_{1}=\left(L_{2} L_{1}^{-1}\right)^{-1} B_{2} L_{2} L_{1}^{-1}$. To conclude, it suffices to apply Lemma 1 below.

Lemma 1. Let $B, B^{\prime}$ be two signings of $A$. If there exists a complex diagonal matrix $\Gamma$ such that $B^{\prime}=\Gamma B \Gamma^{-1}$ and $|\Gamma|=I_{n}$ then $B$ and $B^{\prime}$ are $\{-1,1\}$-diagonally similar. 
Proof. Let $A:=\left(a_{i j}\right)_{1 \leqslant i, j \leqslant n}, B:=\left(b_{i j}\right)_{1 \leqslant i, j \leqslant n}$ and $B^{\prime}:=\left(b_{i j}^{\prime}\right)_{1 \leqslant i, j \leqslant n}$. We denote by $\gamma_{1}, \ldots, \gamma_{n}$ the diagonal entries of $\Gamma$. Let $\Delta:=\gamma_{1}^{-1} \Gamma$. Clearly, we have $\Delta B \Delta^{-1}=\Gamma B \Gamma^{-1}=B^{\prime}$. Hence, to prove our lemma, it suffices to check that $\Delta$ is a $\{-1,1\}$-diagonal matrix. For this, let $j \in\{2, \ldots, n\}$. As $A$ is irreducible, the digraph $D_{A}$ is strongly connected and then there is a path $j=i_{1}, \ldots, i_{r}=1$ of $D_{A}$ from $j$ to 1 . By definition of $D_{A}$, we have $a_{i_{1} i_{2}} \neq 0, \ldots, a_{i_{r-1} i_{r}} \neq 0$. It follows that $b_{i_{1} i_{2}} \neq 0, \ldots, b_{i_{r-1} i_{r}} \neq 0$ and $b_{i_{1} i_{2}}^{\prime} \neq 0, \ldots, b_{i_{r-1} i_{r}}^{\prime} \neq 0$ because $|B|=\left|B^{\prime}\right|=A$. Moreover, from the equality $B^{\prime}=\Gamma B \Gamma^{-1}$ we have $b_{i_{1} i_{2}}^{\prime}=\gamma_{i_{1}} b_{i_{1} i_{2}} \gamma_{i_{2}}^{-1}, b_{i_{2} i_{3}}^{\prime}=\gamma_{i_{2}} b_{i_{2} i_{3}} \gamma_{i_{3}}^{-1}, \ldots$, $b_{i_{r-1} i_{r}}^{\prime}=\gamma_{i_{r-1}} b_{i_{r-1} i_{r}} \gamma_{i_{r}}^{-1}$. Then $b_{i_{1} i_{2}}^{\prime} \ldots b_{i_{r-1} i_{r}}^{\prime}=\gamma_{i_{1}} \gamma_{i_{r}}^{-1} b_{i_{1} i_{2}} \ldots b_{i_{r-1} i_{r}}$. But by hypothesis, $B, B^{\prime}$ are real matrices and $|B|=\left|B^{\prime}\right|$, then $b_{i_{1} i_{2}}^{\prime} \ldots b_{i_{r-1} i_{r}}^{\prime}=$ $\pm b_{i_{1} i_{2}} \ldots b_{i_{r-1} i_{r}}$ and hence $\gamma_{j} \gamma_{1}^{-1}=\gamma_{i_{1}} \gamma_{i_{r}}^{-1} \in\{-1,1\}$, which completes the proof of the lemma.

\section{Proof of the main theorem}

Assertion $i$. (resp. assertion $i i$. for $p=1$ ) follows from Proposition 4 and the fact that $A \in \mathcal{M}(1, A)$ (resp. $-A \in \mathcal{M}(-1, A)$ ). To prove assertion $i$ i. for $p>1$, we will use the cyclic form of irreducible matrices with period $p$. To define $k$-cyclic matrices, let $n$ be a positive integer and let $\left\{r_{1}, \ldots, r_{k}\right\}$ be a partition of $n$, that is $r_{1}, \ldots, r_{k}$ are positive integers and $r_{1}+\cdots+r_{k}=n$. For $i=1, \ldots, k-1$, let $A_{i}$ be a $r_{i} \times r_{i+1}$ matrix and let $A_{k}$ be a $r_{k} \times r_{1}$ matrix. The matrix

$$
\left(\begin{array}{ccccc}
0 & A_{1} & 0 & \cdots & 0 \\
0 & 0 & A_{2} & \cdots & 0 \\
\vdots & \vdots & \ddots & \ddots & \vdots \\
0 & 0 & 0 & \ddots & A_{k-1} \\
A_{k} & 0 & \cdots & 0 & 0
\end{array}\right)
$$

is denoted by $\operatorname{Cyc}\left(A_{1}, A_{2}, \ldots, A_{k}\right)$. Each matrix of this form is called k-cyclic.

The characterization of irreducible matrices with period $p>1$ is given by the following result due to Frobenius.

Proposition 5. Let $A$ be an irreducible nonnegative real matrix with period $p>1$, then there exists a permutation matrix $P$ such that $P A P^{T}$ is p-cyclic. 
Proposition 6. Let $A=\operatorname{Cyc}\left(A_{1}, A_{2}, \ldots, A_{p}\right)$ be a nonnegative $p$-cyclic matrix where $A_{i}$ is a $r_{i} \times r_{i+1}$ matrix for $i=1, \ldots, p-1$ and $A_{p}$ is a $r_{p} \times r_{1}$ matrix. Let $\widetilde{A}$ be the matrix obtained from $A$ by replacing the block $A_{p}$ by $-A_{p}$. Let $k \in\{0, \ldots, 2 p-1\}$, then

i) if $k$ is even, $e^{\frac{i \pi k}{p}} A$ is diagonally similar to $A$, in particular $\operatorname{Sp}(A)=$ $e^{\frac{i \pi k}{p}} \operatorname{Sp}(A)$

ii) if $k$ is odd, $e^{\frac{i \pi k}{p}} A$ is diagonally similar to $\widetilde{A}$, in particular $\operatorname{Sp}(\widetilde{A})=$ $e^{\frac{i \pi k}{p}} \operatorname{Sp}(A)$.

Proof. Let

$$
L:=\left(\begin{array}{ccccc}
I_{r_{1}} & 0 & 0 & \cdots & 0 \\
0 & e^{\frac{i \pi k}{p}} I_{r_{2}} & 0 & \cdots & 0 \\
\vdots & \vdots & \ddots & \ddots & \vdots \\
0 & 0 & 0 & \ddots & 0 \\
0 & 0 & \cdots & 0 & e^{\frac{i \pi k(p-1)}{p}} I_{r_{p}}
\end{array}\right)
$$

It easy to check that if $k$ is even, $e^{\frac{i \pi k}{p}} L A L^{-1}=A$ and if $k$ is odd, $e^{\frac{i \pi k}{p}} L A L^{-1}=\widetilde{A}$.

The next corollary is a consequence of the above proposition and Proposition 5.

Corollary 2. Let $A$ be an irreducible nonnegative matrix with period $p$. Then $\mathcal{M}\left(e^{\frac{i \pi}{p}}, A\right)$ is nonempty.

Proof. As $(-A) \in \mathcal{M}(-1, A)$, we can assume that $p>1$. By Proposition 5, there exists a permutation matrix $P$ such that $P A P^{T}$ is $p$-cyclic. Let $A^{\prime}:=P A P^{T}:=C y c\left(A_{1}^{\prime}, A_{2}^{\prime}, \ldots, A_{p}^{\prime}\right)$ and let $\widetilde{A^{\prime}}$ be the matrix obtained from $A^{\prime}$ by replacing the block $A_{p}^{\prime}$ by $-A_{p}^{\prime}$. It follows from Proposition 6 that $\operatorname{Sp}\left(\widetilde{A^{\prime}}\right)=e^{\frac{i \pi}{p}} \operatorname{Sp}\left(A^{\prime}\right)$, and hence $\operatorname{Sp}\left(P^{T} \widetilde{A^{\prime}} P\right)=e^{\frac{i \pi}{p}} \operatorname{Sp}\left(P^{T} A^{\prime} P\right)=$ $e^{\frac{i \pi}{p}} \operatorname{Sp}(A)$. Let $B_{0}:=P^{T} \widetilde{A^{\prime}} P$. Since $\left|\widetilde{A^{\prime}}\right|=A^{\prime}$, we have $\left|B_{0}\right|=P^{T} A^{\prime} P=$ $A$ and then $B_{0} \in \mathcal{M}\left(e^{\frac{i \pi k}{p}}, A\right)$.

\section{References}

[1] A. Anuradha, R. Balakrishnan, X. Chen, X. Li, H. Lian and W. So, Skew spectra of oriented bipartite graphs, Electron. J. Combin. 20, 2013, \#P18. 
[2] A. Anuradha, R. Balakrishnan and W. So, Skew spectra of graphs without even cycles, Linear Algebra and its Applications, 444, 2014, pp.67-80.

[3] N. Biggs, Algebraic Graph Theory, Second Edition, Cambridge University Press, 1993.

[4] M. Cavers, S.M. Cioaba, S. Fallat, D.A. Gregory, W.H. Haemers, S.J. Kirkland, J.J. McDonald, M. Tsatsomeros, Skew-adjacency matrices of graphs, Linear Algebra Appl. 436, 2012, pp.4512-4529.

[5] D. Cui and Y. Hou, On the skew spectra of Cartesian products of graphs, The Electronic J. Combin. 20 (2), 2013, \#P19.

[6] Y. Hou and T. Lei, Characteristic polynomials of skew-adjacency matrices of oriented graphs, The Electronic Journal of Combinatorics 18, 2011, \#P156.

[7] C.D. Godsil, Algebraic Combinatorics, Chapman and Hall, London, 1993.

[8] B. Shader and W. So, Skew spectra of oriented graphs, The electronic journal of Combinatorics 16, 2009, N 32.

[9] H. Wielandt, Unzerlegbare, nicht negative Matrizen. Mathematische Zeitschrift 52 (1), 1950, pp.642-648.

[10] G. Xu, Some inequalities on the skew-spectral radii of oriented graphs, J. Inequal. Appl. 211, 2012, pp.1-13.

\section{CONTACT INFORMATION}

Kawtar Attas,

Abderrahim

Boussaïri,

Mohamed Zaidi
Laboratoire de Topologie, Algèbre, Géométrie et Mathématiques Discrètes, Faculté des Sciences Aïn Chock, Hassan II University of Casablanca, Casablanca, Morocco.

$$
\begin{array}{r}
E \text {-Mail(s): kawtar.attas@gmail.com, } \\
\text { aboussairi@hotmail.com, } \\
\text { zaidi.fsac@gmail.com }
\end{array}
$$

Received by the editors: 17.09.2019. 